\title{
Equilibrium Conditions at a Solid-Solid Interface
}

\author{
JeeYeon N. Plohr \\ Los Alamos National Laboratory, Theoretical Division, MS-B221, Los Alamos, NM 87545, USA \\ Correspondence should be addressed to JeeYeon N. Plohr, jplohr@lanl.gov
}

Received 22 July 2010; Revised 3 December 2010; Accepted 5 January 2011

Academic Editor: Mohammad Al-Nimr

Copyright (C) 2011 JeeYeon N. Plohr. This is an open access article distributed under the Creative Commons Attribution License, which permits unrestricted use, distribution, and reproduction in any medium, provided the original work is properly cited.

\begin{abstract}
We derive the thermodynamic conditions necessary for two elastoplastic solid phases to coexist in equilibrium. Beyond temperature, velocity, and traction continuity, these conditions require continuity of a generalization of the specific Gibbs free energy. We express this quantity in the Eulerian frame as well as the Lagrangian frame. We also show that two approaches in deriving the equilibrium conditions, one on the continuum level and the other on the atomistic scale, yield the same results. Finally, we discuss two possible interpretations for the Gibbs free energy, which lead to distinct generalizations, except in the case of inviscid fluids, where they coincide.
\end{abstract}

\section{Introduction}

Our goal is to derive the conditions that characterize the thermodynamic equilibrium of two coexisting solid phases for an elastoplastic material. These conditions answer the question: what are the proper boundary conditions at an interface between two solids in equilibrium? This question arises in many applications, such as those involving a transformation from one solid phase to another.

For inviscid fluids, the well-known conditions due to Gibbs provide a complete answer to the corresponding question. For solids, however, only approximate conditions are in widespread use. These approximate conditions are obtained by "extending" the conditions for fluids $[1,2]$. Such extensions are not correct because the behavior of a solid is more complex than that of a fluid, being that the stress in a solid tensor, not simply a scalar.

In the present work, we follow a different approach. We examine the underlying principles that hold at a solid-solid interface, and we reformulate two different approaches to deriving the necessary conditions in the Lagrangian frame. The first approach [3] considers a solid to be a continuum; the second approach [4] views solids on atomistic scale. In reformulating each approach, we avoid assuming that either the transformation strain or the elastic strain is infinitesimal. However, the thickness of the interfacial region is neglected, and surface energy is not considered.
In addition to deriving them for the Lagrangian frame, we derive the equilibrium conditions for the Eulerian frame. In particular, we arrive at a transparent formula for the proper generalization of the Gibbs free energy. Deriving the equilibrium conditions on the continuum level in the Eulerian frame is a prerequisite for comparing the two Lagrangian approaches. We show that these two approaches produce identical thermodynamic equilibrium conditions.

One issue that we highlight is how best to define the Gibbs free energy for solids. We review how the Gibbs free energy for fluids is defined in order to guide the choice of definition for solids. Rather surprisingly, we find that two possible definitions of Gibbs free energy, one as a quantity that must be continuous across a material interface in equilibrium and the other as the chemical potential, yield different formulae for solids.

At the conclusion, we will have a set of necessary conditions for a solid-solid interface to be in equilibrium. In some applications, however, these conditions do not provide a set of boundary conditions that is complete, that is, fully determines the evolution of the interface. We assert that this situation is not a failure for the necessary conditions, but rather is inherent for solid-solid interfaces and that materialspecific interface conditions (e.g., a constitutive relation) are needed as supplemental boundary conditions.

Remark 1. We employ notation that is standard in continuum mechanics instead of that used in $[3,4]$. In rewriting the 
derivations from [4], we use specific quantities (i.e., specific volume, specific energy, etc.) instead of molar densities.

\section{Solid Interface Conditions Derived from the Rankine-Hugoniot Jump Conditions [3]}

The continuum-level thermodynamic equilibrium conditions that hold at an interface between two solids can be derived from the Rankine-Hugoniot jump conditions. These conditions constrain the two states on either side of a discontinuity (which can represent a shock wave or a material interface) in conformance with conservation of mass, momentum, and energy.

2.1. Inviscid Fluid Dynamics. As motivation, we first review the Rankine-Hugoniot jump conditions for inviscid fluid dynamics. Here, $\rho$ is the mass density, $u^{i}$ is the particle velocity, $\varepsilon$ is the specific internal energy, $s$ is the specific entropy, $p$ is the pressure, $v:=\rho^{-1}$ is the specific volume, $q^{i}$ is the specific heat flux, $T$ is the temperature, $s_{n}$ is the interface propagation speed, $m$ is the mass flux through the interface, and $n_{i}$ is the unit vector normal to the interface, pointing from the side labeled - to the side labeled + . Also, for a state quantity $A$, define $\Delta A:=A_{+}-A_{-}$and $\langle A\rangle:=1 / 2\left(A_{-}+A_{+}\right)$. The well-known Rankine-Hugoniot jump conditions corresponding to mass, momentum, and energy conservation are [5-7]

$$
\begin{gathered}
\rho_{-}\left(n_{i} u_{-}^{i}-s_{n}\right)=\rho_{+}\left(n_{i} u_{+}^{i}-s_{n}\right)=: m, \\
m \Delta u+n \Delta p=0, \\
m[\Delta \varepsilon+\langle p\rangle \Delta v]+\Delta\left(n_{i} q^{i}\right)=0 .
\end{gathered}
$$

We also have the entropy inequality that

$$
m \Delta s+\Delta\left(\frac{n_{i} q^{i}}{T}\right) \geq 0
$$

Notice that the mass jump condition implies that

$$
m \Delta v=\Delta\left(n_{i} u^{i}\right)
$$

Remark 2. We allow for heat flow across the interface so that the temperature can become continuous. When the temperature is uniform, the heat flux is zero.

A necessary condition for thermal equilibrium at the interface is that the temperature is continuous. Subtracting the entropy jump condition, multiplied by the common value of temperature, from the energy jump condition gives

$$
m[\Delta(\varepsilon-T s)+\langle p\rangle \Delta v] \leq 0
$$

Since

$$
\begin{aligned}
\langle p\rangle \Delta v & =\Delta(p v)-\langle v\rangle \Delta p \\
& =\Delta(p v)+m\langle v\rangle \Delta\left(n_{i} u^{i}\right) \\
& =\Delta(p v)+m^{2}\langle v\rangle \Delta v \\
& =\Delta(p v)+\frac{1}{2} m^{2} \Delta\left(v^{2}\right),
\end{aligned}
$$

this inequality is equivalent to

$$
m \Delta\left[\varepsilon-T s+p v+\frac{1}{2} m^{2} v^{2}\right] \leq 0 .
$$

In thermodynamic equilibrium, where $m \rightarrow 0$ and no entropy is generated at the interface (i.e., the inequality becomes equality),

$$
\Delta[\varepsilon-T s+p v]=0 .
$$

We recognize the quantity in brackets as the specific Gibbs free energy $g:=\varepsilon-T s+p v$. The thermodynamic equilibrium conditions are therefore

$$
\begin{gathered}
\Delta T=0, \\
\Delta\left(n_{i} u^{i}\right)=0, \\
\Delta p=0, \\
\Delta g=0,
\end{gathered}
$$

where (11) and (12) derive from (5) and (2), respectively, in the limit as $m \rightarrow 0$.

2.2. Lagrangian Elasticity. We turn now to elasticity. In the notation, we employ for Lagrangian continuum mechanics, $F_{\alpha}^{i}$ is the deformation gradient, $\rho_{0}$ is the reference mass density $\left(\rho_{0}=\rho J\right.$, where $\left.J:=\operatorname{det} F\right), V^{i}$ is the particle velocity, $P^{i \beta}$ is the first Piola-Kirchhoff stress tensor, $Q^{\gamma}$ is the specific heat flux, $S_{N}$ is the interface propagation speed, and $N_{\alpha}$ is the vector normal to the interface. The Rankine-Hugoniot jump conditions corresponding to continuity, momentum and energy conservation, and entropy balance are [8]

$$
\begin{gathered}
-S_{N} \Delta F^{i}{ }_{\alpha}=\Delta V^{i} N_{\alpha}, \\
-\rho_{0} S_{N} \Delta V^{i}=\Delta\left(P^{i \beta} N_{\beta}\right), \\
-\rho_{0} S_{N} \Delta \varepsilon=\Delta V_{i}\left\langle P^{i \beta} N_{\beta}\right\rangle-\Delta\left(N_{\gamma} Q^{\gamma}\right), \\
-\rho_{0} S_{N} \Delta S+\Delta\left(\frac{N_{\gamma} Q^{\gamma}}{T}\right) \geq 0 .
\end{gathered}
$$

Remark 3. Because $N_{\alpha}$ and $n_{i}$ are related through $n_{j}=$ $J N_{\beta}\left(F^{-1}\right)^{\beta}$, our assumed normalization $n_{i} n^{i}=1$ precludes setting $N_{\alpha} N^{\alpha}=1$. 
The energy jump condition, combined with the mass jump condition, is

$$
-\rho_{0} S_{N}\left[\Delta \varepsilon-\frac{v_{0}}{N_{\gamma} N^{\gamma}} \Delta\left(F_{i \alpha} N^{\alpha}\right)\left\langle P^{i \beta} N_{\beta}\right\rangle\right]+\Delta\left(N_{\gamma} Q^{\gamma}\right)=0,
$$

where $v_{0}:=\rho_{0}{ }^{-1}$. Subtracting the entropy jump condition, multiplied by the common value of temperature, we obtain

$$
-\rho_{0} S_{N}\left[\Delta(\varepsilon-T s)-\frac{v_{0}}{N_{\gamma} N^{\gamma}} \Delta\left(F_{i \alpha} N^{\alpha}\right)\left\langle P^{i \beta} N_{\beta}\right\rangle\right] \leq 0 .
$$

As

$$
\begin{aligned}
& \Delta\left(F_{i \alpha} N^{\alpha}\right)\left\langle P^{i \beta} N_{\beta}\right\rangle \\
& \quad=\Delta\left(F_{i \alpha} N^{\alpha} P^{i \beta} N_{\beta}\right)-\left\langle F_{i \alpha} N^{\alpha}\right\rangle \Delta\left(P^{i \beta} N_{\beta}\right) \\
& \quad=\Delta\left(F_{i \alpha} N^{\alpha} P^{i \beta} N_{\beta}\right)+\rho_{0} S_{N}\left\langle F_{i \alpha} N^{\alpha}\right\rangle \Delta V^{i} \\
& \quad=\Delta\left(F_{i \alpha} N^{\alpha} P^{i \beta} N_{\beta}\right)-\rho_{0} \frac{S_{N}^{2}}{N_{\gamma} N^{\gamma}}\left\langle F_{i \alpha} N^{\alpha}\right\rangle \Delta\left(F^{i} N^{\beta}\right) \\
& =\Delta\left(F_{i \alpha} N^{\alpha} P^{i \beta} N_{\beta}\right)-\frac{1}{2} \rho_{0} \frac{S_{N}^{2}}{N_{\gamma} N^{\gamma}} \Delta\left(N^{\alpha} C_{\alpha \beta} N^{\beta}\right),
\end{aligned}
$$

where $C_{\alpha \beta}=F_{i \alpha} F_{\beta}^{i}$ is the Cauchy-Green tensor, we find that

$$
\begin{aligned}
-\rho_{0} S_{N} \Delta & {\left[\varepsilon-T s-\frac{1}{N_{\gamma} N^{\gamma}}\right.} \\
& \left.\times\left\{v_{0} F_{i \alpha} N^{\alpha} P^{i \beta} N_{\beta}-\frac{1}{2} \frac{S_{N}^{2}}{N_{\gamma} N^{\gamma}} N^{\alpha} C_{\alpha \beta} N^{\beta}\right\}\right] \leq 0 .
\end{aligned}
$$

In thermodynamic equilibrium, where $S_{N} \rightarrow 0$ and no entropy is generated at the interface,

$$
\Delta\left[\varepsilon-T s-v_{0} \frac{F_{i \alpha} N^{\alpha}}{N_{\gamma} N^{\gamma}} P^{i \beta} N_{\beta}\right]=0 .
$$

As we shall soon justify, we identify the quantity in brackets,

$$
\tilde{g}:=\varepsilon-T s-v_{0} \frac{F_{i \alpha} N^{\alpha}}{N_{\gamma} N^{\gamma}} P^{i \beta} N_{\beta},
$$

as the generalized specific Gibbs free energy. The thermodynamic equilibrium conditions are therefore

$$
\begin{gathered}
\Delta T=0, \\
\Delta V^{i}=0, \\
\Delta\left(P^{i \beta} N_{\beta}\right)=0, \\
\Delta \tilde{g}=0,
\end{gathered}
$$

where (25) and (26) derive from (14) and (15), respectively, in the $S_{N} \rightarrow 0$ limit.
Remark 4. Notice that in contrast to the case of inviscid fluid dynamics, the tangential velocity jump is constrained to be zero; that is, there is no slip at the interface. If the continuity equation is not enforced at the interface, the two materials can slip relative to each other, in a manner controlled, for example, by a friction law.

Define the Eshelby energy-momentum tensor

$$
M_{\alpha}{ }^{\beta}:=\rho_{0}(\varepsilon-T s) \delta_{\alpha}{ }^{\beta}-F_{i \alpha} P^{i \beta} .
$$

Then, the generalized specific Gibbs free energy is related to the normal-normal component of $M_{\alpha}{ }^{\beta}$

$$
\tilde{g}=v_{0} \frac{N^{\alpha} M_{\alpha}^{\beta} N_{\beta}}{N_{\gamma} N^{\gamma}} .
$$

We may also express the Eshelby energy-momentum tensor in terms of the symmetric Piola-Kirchhoff tensor $S^{\alpha \gamma}$, which is defined by the relationship $P^{i \beta}=F_{\alpha}^{i} S^{\alpha \beta}$

$$
M_{\alpha}{ }^{\beta}=\rho_{0}(\varepsilon-T s) \delta_{\alpha}{ }^{\beta}-C_{\alpha \gamma} S^{\gamma \beta} .
$$

When $S^{\alpha \beta}=-J p\left(C^{-1}\right)^{\alpha \beta}$, as it is for an inviscid fluid, the tensor $v_{0} M_{\alpha}{ }^{\beta}$ reduces to $g \delta_{\alpha}{ }^{\beta}$, so that $\tilde{g}$ reduces to $g$. This result motivates calling $\tilde{g}$ the generalized specific Gibbs free energy.

2.3. Eulerian Elasticity. Next, we derive the equivalent form of the thermodynamic equilibrium conditions in the Eulerian frame. Recall that the Cauchy stress is

$$
\sigma^{i j}=J^{-1} F_{\alpha}^{i} S^{\alpha \beta} F_{\beta}^{j}=J^{-1} P^{i \beta} F_{\beta}^{j},
$$

and that the spatial normal $n_{i}$ corresponding to $N_{\gamma}$ is

$$
n_{j}=J N_{\beta}\left(F^{-1}\right)^{\beta} \text {. }
$$

Therefore,

$$
P^{i \beta} N_{\beta}=\sigma^{i j} n_{j} .
$$

Moreover, $F^{i}{ }_{\alpha} N^{\alpha}=J^{-1} b^{i j} n_{j}$ (where $b^{i j}:=F^{i}{ }_{\alpha} F^{j \alpha}$ is the inverse Finger tensor), and $N_{\gamma} N^{\gamma}=J^{-2} n_{p} b^{p q} n_{q}$, so that

$$
v_{0} \frac{F_{i \alpha} N^{\alpha}}{N_{\gamma} N^{\gamma}}=v \frac{n_{k} b^{k}}{n_{p} b^{p q} n_{q}} .
$$

Thus,

$$
\tilde{g}=\varepsilon-T s-v \frac{n_{k} b^{k}}{n_{p} b^{p q} n_{q}} \sigma^{i j} n_{j},
$$

and the thermodynamic equilibrium conditions are

$$
\begin{gathered}
\Delta T=0, \\
\Delta u^{i}=0, \\
\Delta\left(\sigma^{i j} n_{j}\right)=0, \\
\Delta \tilde{g}=0 .
\end{gathered}
$$


Let us introduce the notation

$$
J^{i}:=J \frac{n_{k} b^{k i}}{n_{p} b^{p q} n_{q}}=\frac{F_{\alpha}^{i} N^{\alpha}}{N_{\gamma} N^{\gamma}} .
$$

This notation is natural because the normal component of $J^{i}$ is $J: n_{i} J^{i}=J$. In terms of this notation, the generalized specific Gibbs free energy simplifies to

$$
\tilde{g}=\varepsilon-T s-v_{0} J_{i} \sigma^{i j} n_{j}
$$

and the thermodynamic equilibrium condition is

$$
\Delta\left[\varepsilon-T s-v_{0} J_{i} \sigma^{i j} n_{j}\right]=0 .
$$

When the Cauchy stress is decomposed as

$$
\sigma^{i j}=-p \delta^{i j}+s^{i j}
$$

where $p:=-(1 / 3) \sigma_{k}^{k}$ is the mean pressure and $s^{i j}$ is the deviatoric stress, then the generalized specific Gibbs free energy takes the form

$$
\tilde{g}=\varepsilon-T s+p v-v_{0} J_{i} s^{i j} n_{j}
$$

Thus, the thermodynamic equilibrium condition is

$$
\Delta\left[\varepsilon-T s+p v-v_{0} J_{i} s^{i j} n_{j}\right]=0
$$

If the Cauchy stress is an isotropic pressure, that is, $s^{i j}=0$, then $\tilde{g}$ reduces to $\varepsilon-T s+p v=g$.

More generally, we may derive the approximate form of the generalized specific Gibbs free energy in the small-strain limit. If $\epsilon^{i j}$ denotes the infinitesimal strain, then $b^{i j}$ is related to $\epsilon^{i j}$ through

$$
b^{i j} \approx \delta^{i j}+2 \epsilon^{i j}
$$

to within second order in the displacement gradient. As a result,

$$
v_{0} J^{i} s^{i j} n_{j} \approx v\left[\left(1-2 \epsilon^{n n}\right) s^{n n}+2 n_{i} s^{i j} \epsilon_{j k} n^{k}\right],
$$

where $s^{n n}:=n_{i} s^{i j} n_{j}$ and $\epsilon^{n n}:=n_{i} \epsilon^{i j} n_{j}$. Thus, with $\sigma^{n n}:=$ $n_{i} \sigma^{i j} n_{j}=-p+s^{n n}$

$$
\tilde{g} \approx \varepsilon-T s-\sigma^{n n} v-2 v\left[n_{i} s^{i j} \epsilon_{j k} n^{k}-s^{n n} \epsilon^{n n}\right] .
$$

Notice that the quantity in brackets vanishes if $s^{i j}$ is diagonal.

Remark 5. The generalized Gibbs free energy for solids of Sections 2.2 and 2.3 are based on the conception that the Gibbs free energy is a quantity that is continuous at the phase boundary at the equilibrium. Another interpretation of the Gibbs free energy is that it is the proportionality coefficient between the increase in the total internal energy and the extra mass added to the system, that is, chemical potential. We examine this issue in the discussion.
2.4. Plasticity. Deriving the proper equilibrium conditions from the Rankine-Hugoniot jump conditions that include the effects of plasticity requires the governing equations for the plasticity to be in conservative form. Unlike elasticity, the plastic response of a material depends on the type of a material, the deformation rate, and so forth; a universal form of the plastic flow equation is not available. In this paper, we consider one important class of plasticity, namely, viscoplasticity. In the following, we present the flow rule and hardening law for viscoplasticity in conservative form. We refer the readers to [8] for more details.

First of all, we decompose the deformation gradient as a product

$$
F_{\alpha}^{i}=\left(F_{e}\right)_{a}^{i}\left(F_{p}\right)_{\alpha}^{a},
$$

where $\left(F_{p}\right)^{a \alpha}$, called the plastic part of the deformation gradient, is a matrix of the same type as the gradient of a map from the undeformed (Lagrangian) frame to an intermediate reference frame in which the material is in a stress-free state, and $\left(F_{e}\right)_{a}^{i}$, the elastic part of the deformation gradient, is a matrix of the same type as the gradient of a map from the intermediate reference frame to the deformed (Eulerian) frame. In addition, we define the plastic strain $E^{p} \alpha \beta$ to be

$$
E_{\alpha \beta}^{p}:=\frac{1}{2}\left[\left(F_{p}\right)_{a \alpha}\left(F_{p}\right)_{\beta}^{a}-\delta_{\alpha \beta}\right] .
$$

In general, the flow rule can be written as

$$
\dot{E}_{\alpha \beta}^{p}=\Lambda_{\alpha \beta}\left(E_{\gamma \delta}, E^{p}{ }_{\gamma \delta}, K, S\right),
$$

where $K$ stands for extra internal variables such as a hardening parameter. The hardening law takes the form

$$
\dot{K}=H\left(E_{\gamma \delta}, E^{p}{ }_{\gamma \delta}, K, S\right) .
$$

Both of these equations are in conservative form. The corresponding jump conditions are

$$
\begin{gathered}
-S_{N} \Delta E^{p}{ }_{\alpha \beta}=0, \\
-S_{N} \Delta K=0 .
\end{gathered}
$$

When the interface is moving at the nonzero speed, we conclude that the jump in $E^{p} \alpha \beta$ and $K$ vanish. Taking the $\operatorname{limit} S_{N} \rightarrow 0$, we conclude that

$$
\begin{gathered}
\Delta E^{p}{ }_{\alpha \beta}=0, \\
\Delta K=0,
\end{gathered}
$$

at the interface. Together with (24)-(27), and (54) must be satisfied at the interface between viscoplastic materials in equilibrium.

\section{Thermodynamic Equilibrium across a Coherent Interface [4]}

In this section, we derive the thermodynamic equilibrium conditions across a coherent interface. A coherent transformation in a solid is a phase transformation in which the 


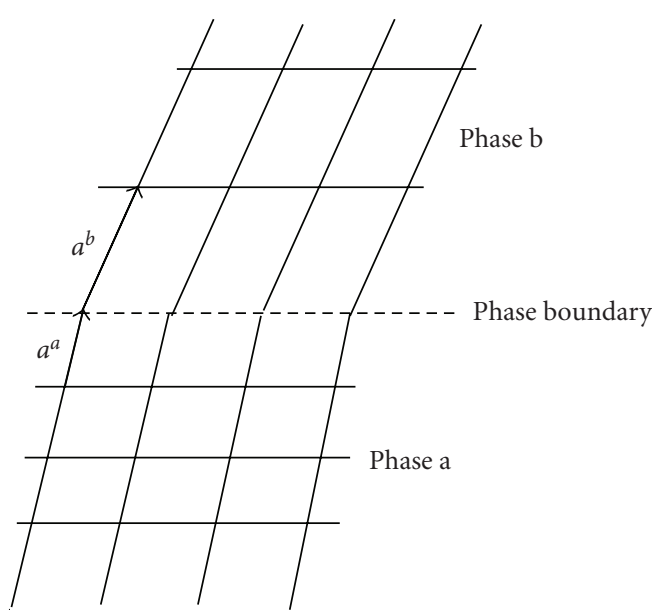

(a)

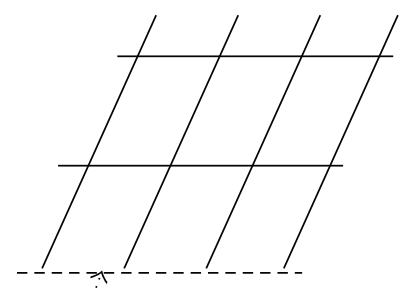

$\delta x^{b}-\delta x^{a}$

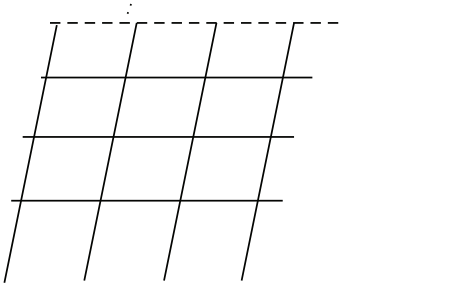

(b)

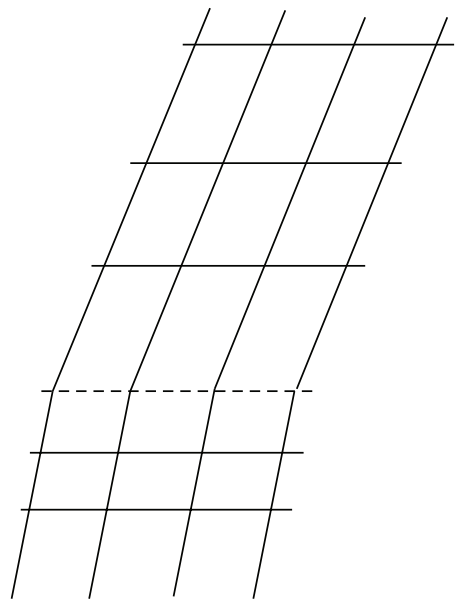

(c)

FIGURE 1: Schematic migration of the phase boundary during a phase transformation.

three-dimensional lattice is preserved without rupture, and a coherent interface is an interface between the different phases of a coherent phase transformation. The treatment here is microscopic; that is, the phase transformation is viewed at the atomic level. In this view, lattice vectors parallel to the interface remain unchanged by the phase transformation.

3.1. Fundamental Equation of State. Let us say that phase $a$ is transformed into phase $b$. Let $x^{i}(i=1,2,3)$ denote the spatial coordinates and $X^{\alpha}(\alpha=1,2,3)$ the reference coordinates. We also use the superscripts $a$ and $b$ to distinguish between the phases $a$ and $b$.

In terms of the deformation gradients $F_{\alpha}^{i}{ }^{a}$ and $F_{\alpha}{ }_{\alpha}^{b}$ of phases $a$ and $b$, the fundamental equations of state of the two phases are

$$
\begin{aligned}
& \varepsilon^{a}=\varepsilon^{a}\left(F^{i}{ }^{a}, s^{a}\right), \\
& \varepsilon^{b}=\varepsilon^{b}\left(F^{i}{ }^{b}{ }^{b}, s^{b}\right) .
\end{aligned}
$$

Infinitesimal variations in the specific internal energies are given by the equations

$$
\begin{aligned}
& \delta \varepsilon^{a}=\left(\frac{\partial \varepsilon^{a}}{\partial F^{i}{ }^{a}}\right) \delta{F^{i}}^{a}{ }^{a}+\left(\frac{\partial \varepsilon^{a}}{\partial s^{a}}\right) \delta s^{a}, \\
& \delta \varepsilon^{b}=\left(\frac{\partial \varepsilon^{b}}{\partial F_{\alpha}^{i}{ }^{b}}\right) \delta F_{\alpha}^{i b}+\left(\frac{\partial \varepsilon^{b}}{\partial s^{b}}\right) \delta s^{b}
\end{aligned}
$$

which can be rewritten as

$$
\begin{aligned}
& \delta \varepsilon^{a}=v_{0} P^{i \alpha a} \delta F_{i \alpha}{ }^{a}+T \delta s^{a}, \\
& \delta \varepsilon^{b}=v_{0} P^{i \alpha b} \delta F_{i \alpha}{ }^{b}+T \delta s^{b} .
\end{aligned}
$$

3.2. Geometry of a Migrating Coherent Interface. The phase transformation of solid can be thought of as the migration of the interface between the two phases. The migration of the interface can be decomposed into the following steps.
(1) An imaginary cut is made along the interface.

(2) Points in $a$ are moved by a distance $\delta x^{i}$, and points in $b$ are moved by a distance $\delta x^{i}$.

(3) A volume of phase $a$ is transformed into phase $b$, reestablishing the contact of the two phases.

These steps, which are illustrated in Figure 1, help in constructing the mathematical formalism, but they are not necessarily physical. Note that only the difference of the displacements $\delta x^{i^{b}}-\delta x^{i^{a}}$ matters and that this vector is determined by the difference between the lattice vectors of the two phases. If phase $b$ has the larger specific volume, the two phases move apart in step (2) so as to make room for phase $a$ to turn into phase $b$ in step (3); otherwise, if phase $a$ has the larger specific volume, parts of the two phases overlap after step (2) to accommodate the shrinkage in step (3).

We can write the relative displacement of $b$ with respect to $a$ as

$$
\delta x^{i^{b}}-\delta x^{i^{a}}=\delta m w^{i},
$$

where $\delta m$ is the mass transformed from phase $a$ to phase $b$ across a unit area of the interface in the reference frame. Thus, $\delta m=n m_{I}$, where $n$ is the number of atomic layers that are transformed and $m_{I}$ is the mass of a single atomic layer at the interface with unit reference are (which is the same for the two phases). We can infer that

$$
w^{i}=\frac{a^{i b}-a^{i a}}{m_{I}},
$$

where $a^{i}$ is a lattice vector in thermodynamic equilibrium that changes during the phase transformation and, therefore, is not parallel to the interface. If the two phases have rectangular crystal structures, $a^{i}$ is the lattice vector that is orthogonal to the interface in the reference state (assuming the reference frame is chosen to be an orthogonal one). 


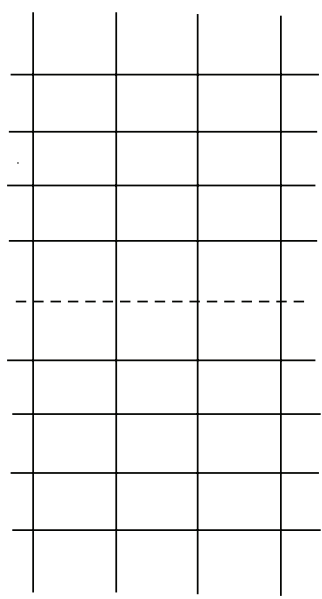

Reference frame

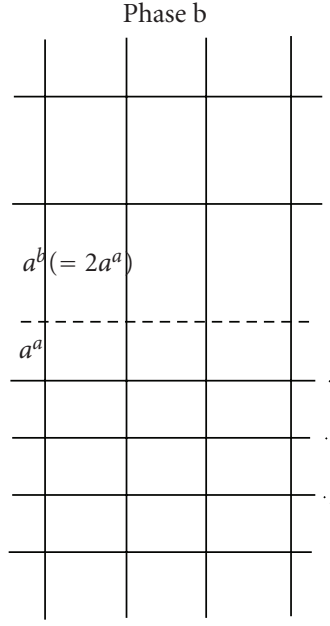

Phase a

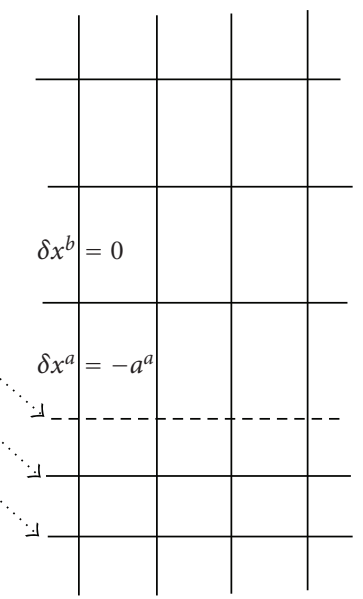

After phase transformation

FIGURE 2: Simple example of a phase transformation.

From these definitions, we find that the interface moves by a distance $\delta \ell$ in the reference frame such that

$$
\delta \ell=\frac{\delta m}{\rho_{0}}=v_{0} \delta m
$$

where $\rho_{0}$ is the reference mass density and $v_{0}$ is the reference specific volume $\left(v_{0}:=\rho_{0}^{-1}\right)$.

As an illustration, we apply the result to a simple case where the lattice structures of two phases are orthogonal, and the lattice constant of phase $b$ is twice longer than that of phase $a$. (Refer to Figure 2.) Let us choose the reference frame that has the same structure as the phase $a$ homogeneously. Suppose that one layer of phase $a$ is transformed into phase $b$. Then, we have the relative displacement as $\delta x^{b}-\delta x^{a}=a^{a}$. On the other hand, $\delta m=m_{I}$ and $w=a^{a} / m_{I}$ so that we have the relation (59). (We have dropped the superscript $i$ labeling a component of a vector, for only the normal component matters in this case.) As only one layer transforms, the distance in the reference frame that the interface moved is $a^{a}$. It is also confirmed from the right-hand side of (61) that $\delta m / \rho_{0}=m_{I} /\left(m_{I} / a^{a}\right)=a^{a}$.

3.3. Conditions of Thermodynamic Equilibrium. Assume that the system comprising phases $a$ and $b$ is in thermodynamic equilibrium. In particular, take the system to be stationary and assume that the temperature is constant throughout the system, since otherwise heat would flow. A fundamental property of a thermodynamic system in thermodynamic equilibrium is that, for an infinitesimal variation away from thermodynamic equilibrium state, with the entropy held fixed, the variation of the energy of the system vanishes

$$
\delta E=0 \text { with entropy fixed, }
$$

where

$$
\delta E=\int_{V_{0}{ }^{a}} \delta \varepsilon^{a} \rho_{0} d V+\int_{V_{0}{ }^{b}} \delta \varepsilon^{b} \rho_{0} d V+\int_{V}\left(\varepsilon^{b}-\varepsilon^{a}\right) \rho_{0} d V .
$$

Here, $V_{0}{ }^{a}$ and $V_{0}{ }^{b}$ are the total volumes of phase $a$ and $b$ in the reference frame, and $V$ is the volume of the region swept by the phase transformation. We proceed to derive conditions for thermodynamic equilibrium from this principle.

Using (58) for the internal energy variation, the total energy variation with the phase transformation becomes

$$
\begin{aligned}
\delta E=\int_{V_{0}{ }^{a}} & \left(v_{0} P^{i \alpha^{a}} \delta F_{i \alpha}{ }^{a}+T \delta s^{a}\right) \rho_{0} d V \\
& +\int_{V_{0}{ }^{b}}\left(v_{0} P^{i \alpha} b F_{i \alpha}{ }^{b}+T \delta s^{b}\right) \rho_{0} d V \\
& +\int_{V}\left(\varepsilon^{b}-\varepsilon^{a}\right) \rho_{0} d V .
\end{aligned}
$$

In the region swept by the phase transformation, the volume element $d V$ can be replaced by $\delta \ell d A$ or $v_{0} \delta m d A$, where $\delta \ell$ and $\delta m$ are defined in the previous section. With this replacement, (64) is rewritten as

$$
\begin{aligned}
\delta E=\int_{V_{0}{ }^{a}}\left(v_{0} P^{i \alpha} \delta F_{i \alpha}{ }^{a}+T \delta s^{a}\right) \rho_{0} d V \\
+\int_{V_{0}{ }^{b}}\left(v_{0} P^{i \alpha} \delta F_{i \alpha}{ }^{b}+T \delta s^{b}\right) \rho_{0} d V \\
+\int_{A}\left(\varepsilon^{b}-\varepsilon^{a}\right) \delta m d A,
\end{aligned}
$$

where $A$ is the area at the interface between two phases.

On the right-hand side of (65), the variations in the displacement and the entropy are not independent. As (62) is valid only when the entropy is held fixed, we impose that

$$
\int_{V_{0}{ }^{a}} \delta s^{a} \rho_{0} d V+\int_{V_{0}{ }^{b}} \delta s^{b} \rho_{0} d V+\int_{A}\left(s^{b}-s^{a}\right) \delta m d A=0 .
$$


We also rewrite the energy variation associated with the displacements by using the following identities:

$$
\begin{aligned}
& \int_{V_{0}{ }^{a}} P^{i \alpha^{a}} \delta F_{i \alpha}{ }^{a} d V \\
& =\int_{A} P^{i \alpha}{ }^{a} N_{\alpha}{ }^{a} \delta x_{i}{ }^{a} d A-\int_{V_{0}{ }^{a}} P_{, \alpha}^{i \alpha}{ }_{, a}^{a} \delta x_{i}{ }^{a} d V
\end{aligned}
$$

and similarly

$$
\begin{aligned}
& \int_{V_{0}{ }^{b}} P^{i \alpha}{ }^{b} \delta F_{i \alpha}{ }^{b} d V \\
& =\int_{A} P^{i \alpha}{ }^{b} N_{\alpha}{ }^{b} \delta x_{i}^{b} d A-\int_{V_{0}{ }^{b}} P_{, \alpha}^{i \alpha}{ }_{, \alpha}^{b} \delta x_{i}^{b} d V,
\end{aligned}
$$

where $N_{\alpha}{ }^{a}$ and $N_{\alpha}{ }^{b}$ are unit vectors normal to the interface in the reference state, drawn outward from $a$ and $b$, respectively, so that $N_{\alpha}{ }^{a}=-N_{\alpha}{ }^{b}$. Strictly speaking, the surface integrals in the first terms of the right hand side in (67) and (68) should be calculated over the whole surface boundaries of phase $a$ and $b$. By assuming that the displacement is negligible on the surface away from the phase boundary (e.g., surrounded by a rigid wall), we reduce the integration area to the interface between two phases, $A$.

Therefore, with the aid of (59), we write the sum of the foregoing two integrals as

$$
\begin{aligned}
\int_{V_{0}{ }^{a}} P^{i \alpha} \delta F_{i \alpha}{ }^{a} d V+\int_{V_{0}{ }^{b}} P^{i \alpha}{ }^{b} \delta F_{i \alpha}{ }^{b} d V \\
=-\int_{A} P^{i \alpha^{a}} N_{\alpha}{ }^{a} w_{i} \delta m d A \\
\quad+\int_{A}\left(P^{i \alpha}{ }^{a} N_{\alpha}{ }^{a}+P^{i \alpha}{ }^{b} N_{\alpha}{ }^{b}\right) \delta x_{i}^{b} d A \\
\quad-\int_{V_{0}{ }^{a}} P^{i \alpha}{ }_{, \alpha}^{a} \delta x_{i}{ }^{a} d V-\int_{V_{0}{ }^{b}} P_{, \alpha}^{i \alpha}{ }^{b} \delta x_{i}^{b} d V .
\end{aligned}
$$

Using (66) and (69), the total energy variation (65) is

$$
\begin{aligned}
\delta E= & -\int_{V_{0}{ }^{a}} P_{, \alpha}^{i \alpha}{ }_{, \alpha}^{a} \delta x_{i}^{a} d V-\int_{V_{0}{ }^{b}} P_{, \alpha}^{i \alpha}{ }^{b} \delta x_{i}^{b} d V \\
& +\int_{A}\left(P^{i \alpha^{a}} N_{\alpha}{ }^{a}+P^{i \alpha^{a}} N_{\alpha}{ }^{b}\right) \delta x_{i}^{b} d A \\
& +\int_{A}\left[\left(\varepsilon^{b}-T s^{b}\right)-\left(\varepsilon^{a}-T s^{a}\right)-w_{i} P^{i \alpha^{a}} N_{\alpha}{ }^{a}\right] \delta m d A .
\end{aligned}
$$

The variational factors in each integral of (70) are independent. The thermodynamic equilibrium condition (62) therefore implies that each integrand is zero.

(1) The volume integrals yield

$$
P_{, \alpha}^{i \alpha}=0 \text { throughout the phases } \mathrm{a} \text { and } \mathrm{b},
$$

which is the usual equation of mechanical equilibrium in the absence of body forces. Equivalently,

$$
\sigma^{i j}{ }_{, j}=0 \quad \text { throughout the phases a and b. }
$$

In deriving (72), we have invoked (31) and the Piola identity, $\left[J\left(F^{-1}\right)^{\alpha}{ }_{i}\right]_{, \alpha}=0$.

(2) The first area integral yields

$$
P^{i \alpha} N_{\alpha}^{a}+P^{i \alpha b} N_{\alpha}^{b}=0
$$

on the interface. Equivalently, by (33),

$$
\sigma^{i j a} n_{j}{ }^{a}+\sigma^{i j b} n_{j}^{b}=0 .
$$

These equations mean that the traction (i.e., the normal stress)

$$
t^{i}:=\sigma^{i j} n_{j}
$$

applied to the interface is continuous, that is, $t^{i^{a}}=t^{i^{b}}$.

(3) Perhaps most importantly, the second area integral yields

$$
\left(\varepsilon^{b}-T s^{b}\right)-\left(\varepsilon^{a}-T s^{a}\right)-w_{i} P^{i \alpha} N_{\alpha}^{a}=0
$$

at the interface. Equivalently,

$$
\left(\varepsilon^{b}-T s^{b}\right)-\left(\varepsilon^{a}-T s^{a}\right)-w_{i} \sigma^{i j} n_{j}=0 .
$$

To understand this condition better, we write $\sigma^{i j}=-p \delta^{i j}+$ $s^{i j}$ and find that

$$
\left(\varepsilon^{b}-T s^{b}\right)-\left(\varepsilon^{a}-T s^{a}\right)+p w^{i} n_{i}-w_{i} s^{i j} n_{j}=0 .
$$

From the definition of $w_{i}$, we derive that

$$
n_{i} w^{i}=n_{i} \frac{\left(a^{i b}-a^{i a}\right)}{m_{I}}=v^{b}-v^{a},
$$

where $v^{a}$ and $v^{b}$ are the specific volumes of phases $a$ and $b$, respectively. Therefore,

$$
\left(\varepsilon^{b}-T s^{b}+p v^{b}\right)-\left(\varepsilon^{a}-T s^{a}+p v^{a}\right)-w_{i} s^{i j} n_{j}=0,
$$

or

$$
g^{b}-g^{a}-w_{i} s^{i j} n_{j}=0,
$$

where $g$ is the usual specific Gibbs free energy. The extra term $w_{i} s^{i j} n_{j}$ accounts for the contribution from the tangential components of the displacement and stress.

\section{Equivalence of the Derived Thermodynamic Equilibrium Conditions}

In this section, we show the equivalence of the two sets of thermodynamic equilibrium conditions that have been derived in Sections 2 and 3.

(a) The temperature continuity condition, (24) or (36), of Section 2 corresponds to the somewhat stronger condition of temperature constancy assumed in Section 3 . 
(b) The velocity continuity condition, (25) or (37), of Section 2 corresponds to the somewhat stronger condition of stationarity in Section 3.

(c) The traction continuity conditions (26) and (73) involving the Piola-Kirchhoff stress are identical, as are the corresponding conditions (38) and (74) involving the Cauchy stress.

(d) We compare (42) and (77) under the assumption that the phase transformation is coherent. Evidently, these equations are equivalent if we show that

$$
w^{i}=v_{0} \Delta J^{i} .
$$

By the coherence of the phase transformation, there exists a lattice vector $a_{0}^{\alpha}$ in the reference frame that maps, under $F_{\alpha}^{i}{ }^{a}$ and $F_{\alpha}^{i}{ }^{b}$, to the spatial lattice vectors $a^{i a}$ and $a^{i b}$, respectively. By definition of $w^{i}$,

$$
w^{i}=\frac{a^{i b}-a^{i a}}{m_{I}}=\frac{\Delta F_{\alpha}^{i} a_{0}^{\alpha}}{m_{I}} .
$$

Recall that the deformation gradient $F_{\alpha}^{i}:=\partial x^{i} / \partial X^{\alpha}$ satisfies

$$
\epsilon^{\alpha \beta \gamma} F_{\beta, \gamma}^{i}=0 \text {, }
$$

when it is smooth and satisfies the analogous jump conditions

$$
\epsilon^{\alpha \beta \gamma} \Delta F_{\beta}^{i} N_{\gamma}=0
$$

at discontinuities. In other words, $\Delta F^{i}{ }_{\alpha} \propto N_{\alpha}$. The definition of $J^{i}:=F^{i}{ }_{\alpha} N^{\alpha} /\left(N_{\gamma} N^{\gamma}\right)$ determines the proportionality factor

$$
\Delta F_{\alpha}^{i}=\Delta J^{i} N_{\alpha}
$$

As a result,

$$
w^{i}=\frac{\Delta J^{i} N_{\alpha} a_{0}^{\alpha}}{m_{I}}=v_{0} \Delta J^{i}
$$

\section{Summary and Discussion}

5.1. Equilibrium Conditions. In the subject of solid-solid phase transformations, the question-what are the correct thermodynamic equilibrium conditions between solid phases?-has not been answered satisfactorily. For inviscid fluids, the answer is continuity of (a) temperature, (b) velocity, (c) pressure, and (d) specific Gibbs free energy. The situation is more complicated for solids. The first issue concerns properly generalizing the Gibbs free energy when the stress tensor is not simply a pressure. Certainly, the term replacing $p v$ in the usual Gibbs free energy should reduce to $p v$ when the stress tensor is $\sigma^{i j}=-p \delta^{i j}$. Yet, there are many combinations of stress, strain, and specific volume that satisfy this criterion. The second issue is whether there are enough conditions to determine the states of the two phases.
We have derived the following thermodynamic equilibrium conditions at the interface between two solid phases:

(1) continuity of the temperature,

(2) continuity of the velocity,

(3) continuity of the traction,

(4) continuity of the generalized specific Gibbs free energy.

We assert that these results address the two issues mentioned above.

In constructing the thermodynamic equilibrium conditions for two solid phases, we have paid close attention to the origin of these conditions for the case of fluids. The fluid conditions are so physically intuitive that we often take them for granted. However, we recognize that the underlying physical principles are the conservation laws and the entropy inequality. These principles generate a complete set of conditions connecting the two phases. For fluids, these principles yield the commonly accepted thermodynamic equilibrium conditions.

For solids, we have derived the corresponding conditions in Section 2. The derivation yields a particular generalization of the specific Gibbs free energy

$$
g=\varepsilon-T s+p v
$$

is generalized to

$$
\tilde{g}=\varepsilon-T s+p v-v_{0} J_{i} s^{i j} n_{j} .
$$

Perhaps surprisingly, the generalized specific Gibbs free energy is independent of the tangential-tangential components of stress. Because no thermodynamic equilibrium conditions involve stress components other than the tractions $\left(t^{i}=\sigma^{i j} n_{j}\right)$, it might be thought that our derivation is incomplete. Nonetheless, the conditions that we derived are necessary and sufficient to conserve mass, momentum, and energy and to satisfy the entropy inequality. If these are all of the physical principles we require to be satisfied between two phases, these conditions are all we get. We conclude that any further conditions, if needed, must be materials-specific constitutive relations.

For example, these further conditions might specify the interfacial interaction between the phases. Do they slip freely past each other, or are they fixed together? Such a question, the answer to which reflects a material property, lies outside the scope of general thermodynamic arguments.

Remark 6. A similar issue arises in any physical problem that treats an interface. In interfacial instability problems, like Rayleigh-Taylor or Richtmyer-Meshkov flows for solids, modeling requires assumptions concerning the properties of the material interface $[9,10]$.

We emphasize that the derivation in Section 3 makes stronger assumptions (global thermodynamic equilibrium) and applies only to a very special type of phase transformation, a coherent transformation. In general, a phase transformation can link very different crystal structures, which might 
not be coherent. Even leaving aside melting and gasification, many types of phase transformation between solid phases are not coherent. Thus, the derivation in Section 2 is more general. Nonetheless, the detailed picture in Section 3 helps clarify the physics behind the thermodynamic equilibrium condition.

5.2. Gibbs Free Energy. Before closing the discussion, we want to bring your attention to the interpretation of the Gibbs free energy. The generalized Gibbs free energy defined in (89) is interpreted as a quantity that should be continuous at the phase interface at the equilibrium (chemical potential). On the other hand, the Gibbs free energy can also be viewed as an increase in total internal energy with the addition of a unit mass to the thermodynamic system. In this section, we derive the Gibbs free energy based on the latter notion to find the result different from (89). For concreteness of the argument, we assume the thermoelasticity.

The equation of state for a thermoelastic material is

$$
u=u\left(s, E_{\alpha \beta}\right) \text {, }
$$

where $u$ is specific internal energy, $s$ is specific entropy and $E_{\alpha \beta}$ is Cauchy-Green Lagrangian strain tensor. Then, the fundamental thermodynamic identity for thermoelastic solids is

$$
\rho d u=\rho T d s+J^{-1} S^{\alpha \beta} d E_{\beta \alpha},
$$

where $S^{\alpha \beta}:=\rho_{0} \partial u / \partial E_{\alpha \beta}$ is the symmetric Piola-Kirchhoff stress tensor and $J:=(\operatorname{det} C)^{1 / 2}$ is the Jacobian of $F$, which is such that $\rho=J^{-1} \rho_{0}$. From (31), $\sigma^{i j}=J^{-1} F^{i}{ }_{\alpha} S^{\alpha \beta} F^{\beta}{ }_{j}$.

To better understand the work term $J^{-1} S^{\alpha \beta} d E_{\beta \alpha}$ in (91), we decompose the strain tensor $E$ into the volumetric part and shear part. Let

$$
\bar{C}_{\alpha \beta}:=J^{-2 / 3} C_{\alpha \beta}, \quad \bar{E}_{\alpha \beta}:=\frac{1}{2}\left(\bar{C}_{\alpha \beta}-\delta^{\alpha \beta}\right) .
$$

Note that $\operatorname{det} \bar{C}=1$, that is, $\bar{C}$ is a shear strain.

Using these definitions, the differential of $E_{\alpha \beta}$ is

$$
d E_{\alpha \beta}=\frac{1}{3} J^{-1} C_{\alpha \beta} d J+J^{2 / 3} d \bar{E}_{\alpha \beta}
$$

and therefore,

$$
J^{-1} S^{\alpha \beta} d E_{\beta \alpha}=-J^{-1} \bar{p} d J+J^{-1 / 3} S^{\alpha \beta} d \bar{E}_{\beta \alpha},
$$

where $\bar{p}:=-1 / 3 \operatorname{tr} \sigma$ is the mean pressure.

In terms of the Cauchy stress, this equation becomes

$$
\begin{aligned}
& J^{-1} S^{\alpha \beta} d E_{\beta \alpha} \\
& \quad=-J^{-1} \bar{p} d J+J^{2 / 3}\left[\left(F^{-1}\right)^{\alpha}{ }_{i}(\operatorname{dev} \sigma)^{i j}\left(F^{-T}\right)_{j}^{\beta}\right] d \bar{E}_{\beta \alpha},
\end{aligned}
$$

by observing that $\left(C^{-1}\right)^{\alpha \beta} d \bar{E}_{\beta \alpha}=0$ so that $S^{\alpha \beta}$ on the righthand side in (94) can be replaced by $S^{\alpha \beta}-1 / 3 S^{\gamma \delta} C_{\delta \gamma}\left(C^{-1}\right)^{\alpha \beta}$, which equals $J\left(F^{-1}\right)^{\alpha}{ }_{i}(\operatorname{dev} \sigma)^{i j}\left(F^{-T}\right)^{j}{ }_{\beta}$.
Hence, we get the fundamental thermodynamic identity in Eulerian frame

$$
\rho d u=\rho T d s-\rho \bar{p} d v+J^{2 / 3}(\operatorname{dev} \sigma)^{i j}\left(F^{-T}\right)_{j}^{\alpha} d \bar{E}_{\alpha \beta}\left(F^{-1}\right)^{\beta}{ }_{i} .
$$

Now, note that an external quantity $Q$ of thermodynamic system satisfies $Q=m q$, where $m$ is total mass of the system and $q$ is corresponding specific quantity of $Q$. Hence, we write the internal energy, entropy, and volume as

$$
\begin{gathered}
U(\text { total internal energy })=m u, \\
S(\text { total entropy })=m s, \\
V(\text { total volume })=m v .
\end{gathered}
$$

Then, the fundamental thermodynamic identity (96), multiplied by $V$ is

$$
\begin{aligned}
d U-u d m= & T(d S-s d m)-\bar{p}(d V-v d m) \\
& +V J^{2 / 3}(\sigma)^{i j}\left(F^{-T}\right)_{j}^{\alpha} d \bar{E}_{\alpha \beta}\left(F^{-1}\right)_{i}^{\beta},
\end{aligned}
$$

so that

$$
\begin{aligned}
d U= & T d S-\bar{p} d V+V J^{2 / 3}(\sigma)^{i j}\left(F^{-T}\right)_{j}^{\alpha} d \bar{E}_{\alpha \beta}\left(F^{-1}\right)_{i}^{\beta} \\
& +(u-T s+\bar{p} v) d m .
\end{aligned}
$$

Thus, we are led to identify the coefficient of $d m$ as the specific Gibb free energy

$$
g:=-\left.T \frac{\partial U}{\partial m}\right|_{S, V, \bar{E}}=u-T s+\bar{p} v
$$

Note that there is no tensorial dependency and instead only pressure and volume enter the formula in contrast to (89). For fluids, the two interpretations yield the same definition.

\section{References}

[1] B. E. Clements, J. N. Plohr, and F. L. Addessio, "Dynamically driven phase transitions in composites: I. Basic theoryand microstructure considerations," Journal of Applied Physics, vol. 100, no. 12, Article ID 123520, 2006.

[2] J. N. Plohr, B. E. Clements, and F. L. Addessio, "Dynamically driven phase transitions in composites: II. Applicationsincluding damage," Journal of Applied Physics, vol. 100, no. 12, Article ID 123521, 2006.

[3] K. Wilmański, Thermomechanics of Continua, Springer, Berlin, Germany, 1998.

[4] P.-Y. Robin, "Thermodynamic equilibrium across a coherent interface in a stressed crystal," American Mineralogist, vol. 59, pp. 1286-1298, 1974.

[5] J. Smoller, Shock Waves and Reaction-Diffusion Equations, Springer, New York, NY, USA, 1982.

[6] J. D. Anderson, Modern Compressible Flow with Historical Perspective, McGraw-Hill, New York, NY, USA, 1990.

[7] R. J. LeVeque, Numerical Methods for Conservation Laws, Birkhäuser, Basel, Switzerland, 1994. 
[8] B. J. Plohr and D. H. Sharp, "A conservative formulation for plasticity," Advances in Applied Mathematics, vol. 13, no. 4, pp. 462-493, 1992.

[9] B. J. Plohr and D. H. Sharp, "Instability of accelerated elastic metal plates," Zeitschrift fur Angewandte Mathematik und Physik, vol. 49, no. 5, pp. 786-804, 1998.

[10] J. N. Plohr and B. J. Plohr, "Linearized analysis of RichtmyerMeshkov flow for elastic materials," Journal of Fluid Mechanics, vol. 537, pp. 55-89, 2005. 

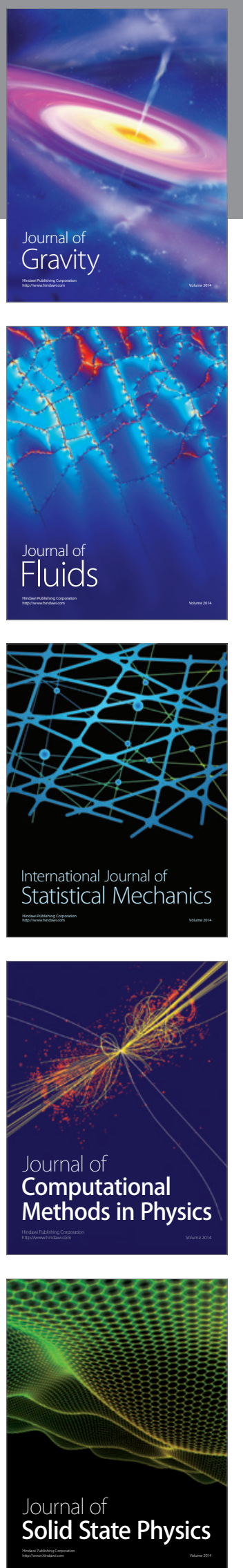

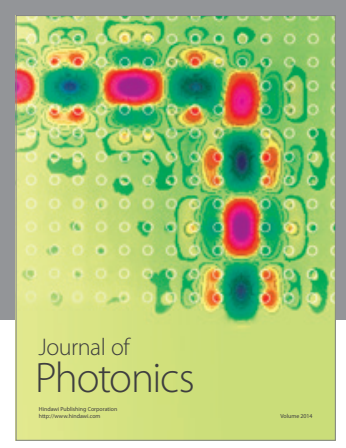

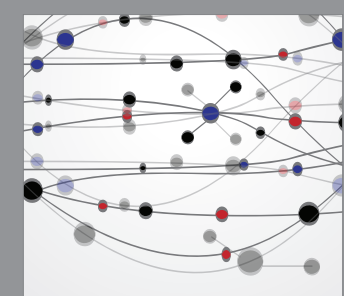

The Scientific World Journal
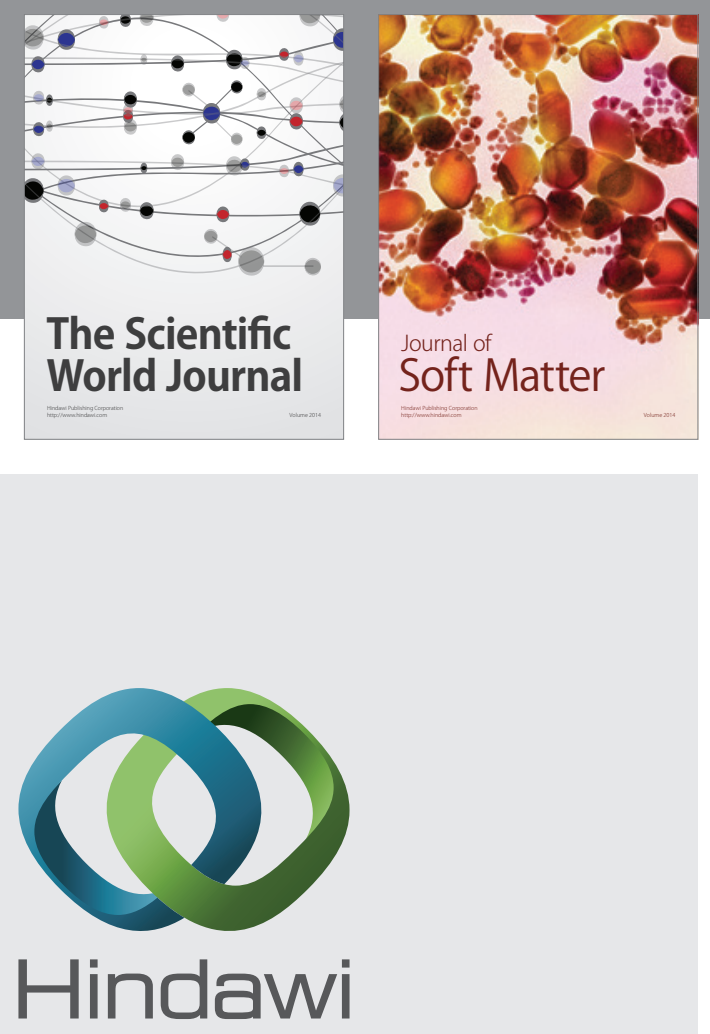

Submit your manuscripts at

http://www.hindawi.com
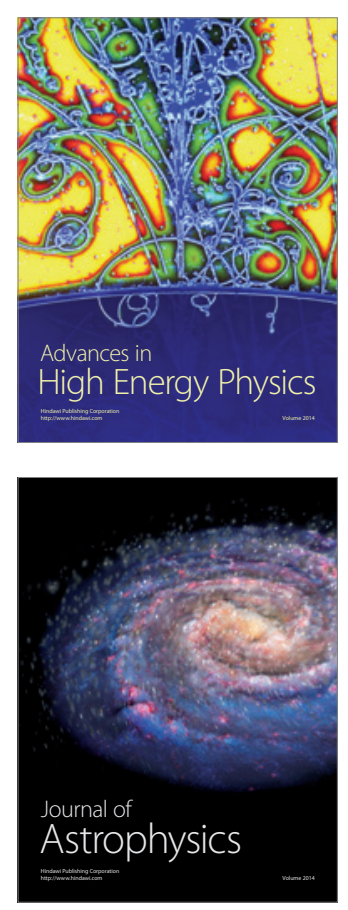
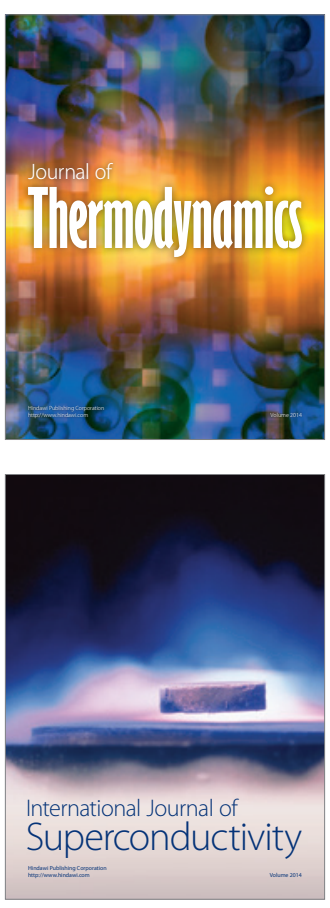
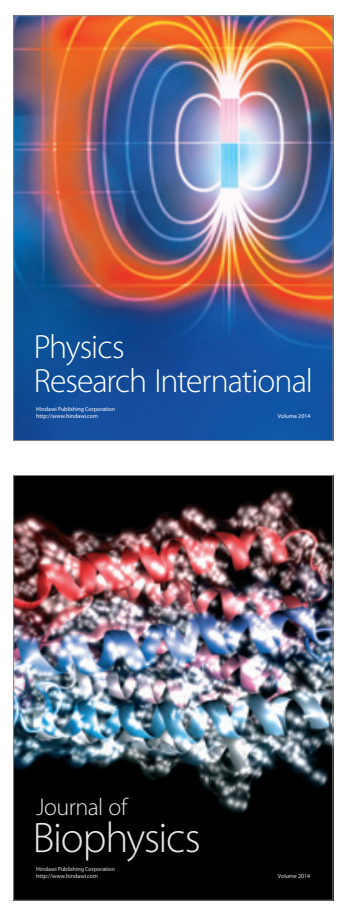
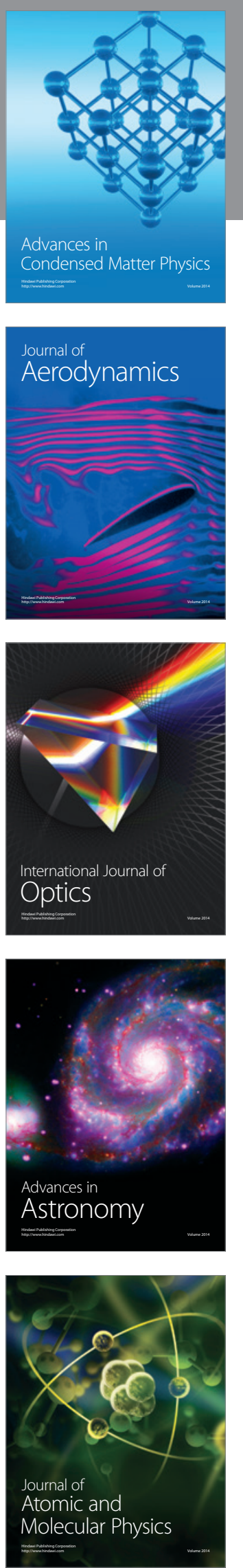